\title{
The Role of Nephrectomy of the Atrophic Kidney in Bearers of Renovascular Hypertension
}

\author{
Myrian J. Thomaz, Antonio M. Lucon, Jose N. Praxedes, Luis A. Bortolotto, Miguel Srougi \\ Department of Urology, Faculty of Medicine, University of Sao Paulo, Sao Paulo, Brazil
}

\begin{abstract}
Purpose: Evaluation of the beneficial effect of nephrectomy of the atrophic kidney on blood pressure (BP) and renal function.

Materials and Methods: A retrospective study of 51 patients with renovascular hypertension (RVH), bearers of atrophic kidney due to severe stenosis or occlusion of the renal artery. Average age was $47.1 \pm 15$ years, the median creatinine clearance was $54 \mathrm{~mL} / \mathrm{min}$, average systolic BP (SBP) $149.6 \pm 22.5 \mathrm{~mm} \mathrm{Hg}$, average diastolic BP (DBP) $90.8 \pm 17 \mathrm{~mm} \mathrm{Hg}$ and the median number of hypotensors 3 ( 1 to 5 ) per patient per day. Blood pressure and serum creatinine were analyzed from 12 to 60 months after the nephrectomy.

Results: There was a significant improvement in the average SBP in the periods from 12 to 36 months $(\mathrm{p} \leq 0.028)$ and for the average DBP from 12 to 48 months after the nephrectomy ( $\mathrm{p} \leq 0.045)$, accompanied by a significant reduction in the use of hypotensors from 12 to 48 months $(\mathrm{p}<0.05)$. One year after the nephrectomy, there was a $69 \%$ improvement in blood pressure and $63.8 \%$ improvement in renal function of patients.

Conclusion: The removal of atrophic kidney in patients with RVH is a safe procedure which presents benefits for the control of arterial hypertension and renal function in bearers of renovascular hypertension.
\end{abstract}

Key words: nephrectomy; atrophic; kidney renovascular; hypertension

Int Braz J Urol. 2010; 36: 159-70

\section{INTRODUCTION}

Among the causes of secondary hypertension, we emphasize renovascular hypertension resulting from renal ischemia caused by the total or partial stenosis of the renal artery, presenting an atherosclerotic etiology in $90 \%$ of the cases and fibrodysplasia of the artery in $10 \%(1,2)$. It is the second most common cause of secondary hypertension, only after parenchymal renal diseases. It occurs in from 1 to $5 \%$ of the adult hypertensive population, but increases in the elderly, diabetics and bearers of systemic atheroscle- rotic lesions $(1,3)$. It has growing participation as a cause of dialytic renal insufficiency $(4,5)$.

Ischemic nephropathy is caused by the chronic and hemodynamically significant occlusion of the renal artery which results in a considerable reduction in the blood flow, a diminution in the glomerular filtration rate and leads to the progressive damage of the renal parenchyma $(1,3,6)$. The renal hypoflow results in the activation of the renin-angiotensin-aldosterone (RAA) system, responsible in the final analysis for vascular alterations, damage to the dependent endothelial vasodilation and an increase in oxidative stress, 
mainly in the etiology of atherosclerotic patients (7).

Renovascular hypertension (RVH) can be cured in special cases. The revascularization of the ischemic kidney, whether surgical or by percutaneous angioplasty (PTA), is the best means of treatment and should be considered when pharmacological treatment is insufficient for the adequate control of the blood pressure or when inexplicable renal insufficiency appears. We analyzed the nephrectomy of the atrophic kidney (less than $8 \mathrm{~cm}$ ) in those patients with refractory blood pressure or a deterioration of the renal function, when the revascularization of the artery was no longer possible.

\section{MATERIALS AND METHODS}

This was a retrospective observational study of 61 patients with a diagnosis of RVH who underwent nephrectomy during the period from January 1989 to January 2007. Ten patients were lost to follow-up. The general data of the patients is found in Table-1. The average age of the patients was $47.1 \pm$ 15 years with a predominance of the female sex and white. The average pre-operative SBP was $149.6 \pm$ $22 \mathrm{~mm} \mathrm{Hg}$ and the average pre-operative diastolic blood pressure (DBP) was $90.8 \pm 16.7 \mathrm{~mm} \mathrm{Hg}$ with $2.8 \pm 1$ hypotensors per patient per day. The median pre-operative serum creatinine was $1.3 \mathrm{mg} / \mathrm{dL}(0.8$ to $4.5 \mathrm{mg} / \mathrm{dL}$ ) analyzed by the colorimetric method (8). Hypercholesterolemia and smoking were the most frequent risk factors.

The average renal size of the atrophic kidney was $7.1 \pm 1.1 \mathrm{~cm}(10$ to $5.2 \mathrm{~cm})$ and the contralateral kidney was $10.9 \pm 0.9 \mathrm{~cm}(9.3$ to $13.5 \mathrm{~cm})$, measured by ultrasonography. On echo-Doppler examination of the renal arteries the stenosis which made up $\geq 70 \%$ of the diameter of the blood vessel were considered as hemodynamically significant lesions. The function of the atrophic kidney measured by renal scintigraphy with the radioisotope DTPA and captopril was $8 \%$ $(0-49 \%)$.

The measurement of the plasma rennin activity (PRA) was performed by radioimmunoassay Gamma Coat PRA I125 Ria Kit, by the collection of blood by selective catheterization of the renal veins and cava inferior.

Of the $35 \%(18 / 51)$ of patients who were bearers of significant bilateral lesion, $17.6 \%(9 / 51)$

Table 1 - General data of the 51 patients with renovascular hypertension who underwent nephrectomy.

\begin{tabular}{lc}
\hline Average age (years $\pm \mathrm{SD}$, minimum - maximum) & $47 \pm 15(13-77)$ \\
Sex: female / male & $67 \% / 33 \%$ \\
Color: white / non-white & $71 \% / 29 \%$ \\
Period of arterial hypertension (years - median) & $11.5(1$ month - 30 years) \\
Average systolic blood pressure in mm Hg & $149.6 \pm 22.5$ \\
Average diastolic blood pressure in mm Hg & $90.8 \pm 16.7$ \\
No. of hypotensor drugs / patient / day & $2.8 \pm 1.0$ \\
Pre-operative (median) serum creatinine mg/dL & $1.3(0.8-4.4)$ \\
Hypercholesterolemia & $67 \%$ \\
Smoker and ex-smoker & $53 \%$ \\
Diabetes mellitus & $18 \%$ \\
Previous cardiac disease & $31 \%$ \\
Previous cerebrovascular disease & $21 \%$ \\
Peripheral vascular disease / aortic aneurism & $19 \%$ \\
\hline
\end{tabular}


required revascularization by percutaneous transluminal angioplasty (PTA) of the contralateral artery. The previous surgical revascularization by means of an autotransplant of the contralateral kidney was carried-out in $9.8 \%(5 / 51)$ of the patients, $4 \%(2 / 51)$ of whom no longer required dialysis. Eight percent (4/51) did not undergo any procedure.

The arteriography, performed by means of selective catheterization of the renal arteries, was performed in 74\% (38/51) of the cases. The magnetic resonance angiography of the renal arteries was performed in 16\% (8/51) of the patients and its principal indicator was the presence of creatinine equal to or greater than $2 \mathrm{mg} / \mathrm{dL}$.

The recommendation for the nephrectomy of the atrophic kidney was made only after total or significant occlusion of the renal artery, associated with the absence of renal function, had been demonstrated. In $70.5 \%$ of the patients nephrectomies by video laparoscopy were performed and in the remaining $29.5 \%$ nephrectomies by lumbotomy.

The postoperative measurements of blood pressure were made in accordance with the recommendations of the WHO. and were completed each 12 months for five years, with the same period for the collection of serum creatinine. Patients were regarded as cured who had DBP $<90 \mathrm{~mm} \mathrm{Hg}$ and systolic (SBP) $<140 \mathrm{~mm} \mathrm{Hg}$ with no hypotensor medication and with an improvement in blood pressure with $\mathrm{DBP}<90 \mathrm{~mm}$ $\mathrm{Hg}$ and /or SBP $<140 \mathrm{~mm} \mathrm{Hg}$ with the same or a reduction in hypotensor medication or with a reduction in DBP of at least $15 \mathrm{~mm} \mathrm{Hg}$ with the same number or a reduction in hypotensor medication (9). We considered therapeutic failures those cases in which there was a worsening of blood pressure or an increase in the number of hypotensors for the same pressure levels; all the other cases benefited from the treatment, in accordance with the criteria cited above (9).

In the assessment of renal function, we used the estimated creatinine clearance, obtained on the basis of the MDRD equation (modification of diet in renal disease study prediction equation) $(9,10)$. The numbers obtained were transferred to the creatinine clearance graph where each line represents a patient throughout the period of observation, the horizontal and ascending lines indicating an improving and the descending ones, a worsening renal function.

\section{Statistical Analysis}

The data have been summarized as numbers and percentages for the qualitative variables and as averages \pm standard deviations where the supposition of normality of the data has been satisfied and in those cases for which this supposition could not be made, the median was used (minimum - maximum).

The assessment of the pressure levels over the study period was performed by means of a variance analysis model (ANOVA) and the measurements repeated, the multiple comparisons were submitted to the construction of contrasts.

The comparison between the groups ( $\mathrm{R} \times \mathrm{NR}$ ) (Responders $\mathrm{x}$ Non-Responders) or Improvement $\mathrm{x}$ Worsening) was made by means of the Chi-squared test when the variable was qualitative and in those cases in which the value expected was less than five, the Fisher's Exact Test was used. For the quantitative variables Student's-t-test or Mann's test was used for those cases in which the supposition of normality could not be made.

A significance level of 5\% was considered significant. SAS (Statistical Analysis System V. 9.0) software was used for the analysis of the data.

\section{RESULTS}

There was statistically significant variation both for the SBP $(p<0.001)$ and for the DBP $(p=$ 0.005 ) over time (Figure-1). The decrease observed at 12 months post nephrectomy was $14.5 \pm 2.8 \mathrm{~mm}$ $\mathrm{Hg}(\mathrm{p}<0.001)$ for the SBP and $9.7 \pm 2.4 \mathrm{~mm} \mathrm{Hg}$ $(\mathrm{p}<0.001)$ for DBP. From 12 months to 60 months postoperatively there was no statistically significant variation whether in SBP $(\mathrm{p}>0.05)$ or $\mathrm{DBP}(\mathrm{p}>$ $0.05)$, shown in the respective Tables- 2 and 3. It was observed that the average decrease in SBP from the pre-nephrectomy period to each of the periods assessed was significant until 36 months post-nephrectomy, while the average decrease in the DBP presented statistical significance until 48 months as compared with that of the pre-nephritic period (Table-4).

The average number of hypotensors used in the pre-operative period of 2.8 hypotensors/day diminished significantly $(\mathrm{p}<0.05)$ for the period 


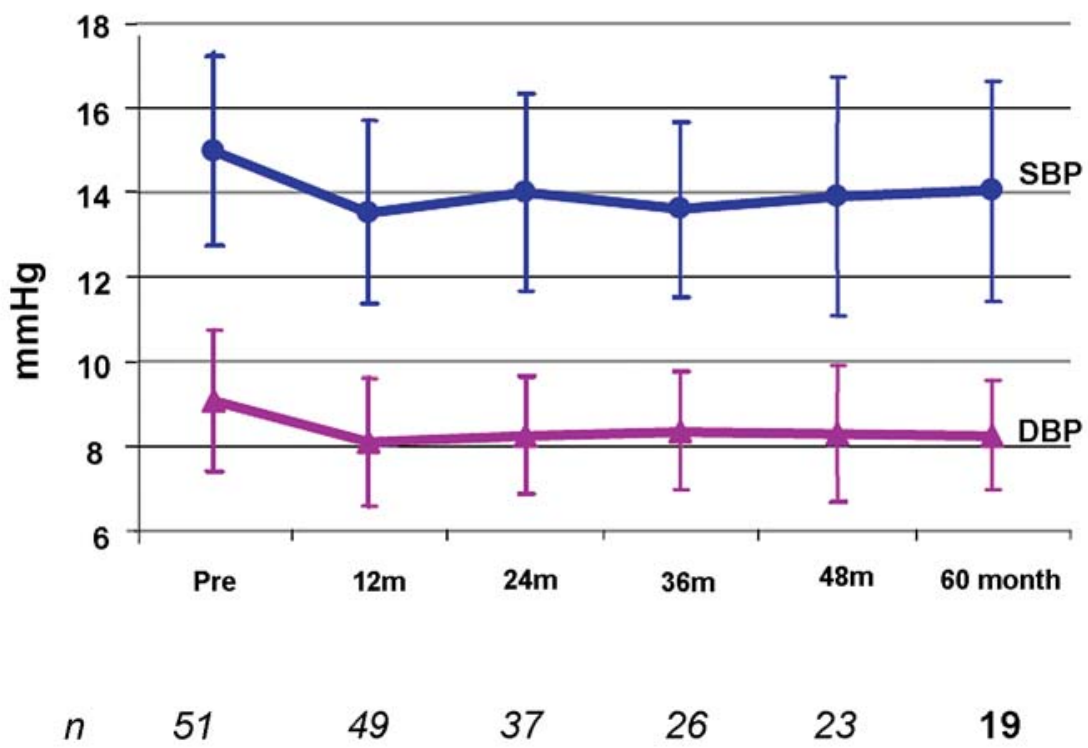

Figure 1 - Average SBP and DBP pre - and post-nephrectomy in patients with RVH.

of 12 months and remained constant until 48m (2.0 hypotensors/day) after the nephrectomy.

One year after the nephrectomy 49 patients were still being followed-up and the BP of $69 \%$ of them had improved, in accordance with the criteria previously mentioned (9). There was one death and one dialytic renal insufficiency $(2 / 51)$, which were excluded from the sample. The value of the variations of the DBP and SBP at 12 months for each patient are shown in Figures-2 and 3 respectively, where in the lower part of each figure are shown the patients whose pressure levels improved. There was an 8\% (4/51) cure rate of RVH, 3 of the patients had fibromuscular dysplasia of the renal artery.

Sixty-nine percent (34/49) of the patients were considered responders as they had shown an improvement in blood pressure and 30.6\% (15/49) of the patients as non-responders as their blood pressure had worsened. There was no significant difference $(p>0.05)$ between the groups for any of the factors analyzed: age, sex, color, initial blood pressure, initial creatinine, bilateral disease, risk factors and co-morbidities (Table-5). The renal function of the patients was checked by the measurement of serum

Table 2 - Averages of the systolic blood pressures from the pre-operative period until 60 months pos-nephrectomy.

\begin{tabular}{lcccccc}
\hline $\mathbf{m m} \mathbf{H g}$ & Pre & $\mathbf{1 2} \mathbf{~ m}$ & $\mathbf{2 4} \mathbf{~ m}$ & $\mathbf{3 6} \mathbf{~ m}$ & $\mathbf{4 8} \mathbf{~ m}$ & $\mathbf{6 0} \mathbf{~ m}$ \\
\hline Average & 149.6 & 135.2 & 140.0 & 136.0 & 139.0 & 140.4 \\
DP & 22.5 & 21.8 & 23.3 & 20.6 & 28.3 & 26.1 \\
Median & 140.0 & 136.0 & 134.0 & 132.5 & 132.0 & 130.0 \\
Minimum & 110.0 & 100.0 & 100.0 & 100.0 & 105.0 & 110.0 \\
Maximum & 210.0 & 190.0 & 186.0 & 176.0 & 220.0 & 200.0 \\
No. observed & 51 & 49 & 37 & 26 & 23 & 19 \\
\hline
\end{tabular}


Table 3 - Averages of the diastolic blood pressures (DBP) from the pre-operative period until 60 months pos-nephrectomy.

\begin{tabular}{lcccccc}
\hline $\mathbf{m m} \mathbf{H g}$ & $\mathbf{P r e}$ & $\mathbf{1 2} \mathbf{~}$ & $\mathbf{2 4} \mathbf{~}$ & $\mathbf{3 6} \mathbf{~}$ & $\mathbf{4 8} \mathbf{~ m}$ & $\mathbf{6 0} \mathbf{~}$ \\
\hline Average & 90.8 & 81.1 & 82.7 & 83.6 & 83.0 & 82.6 \\
DBP & 16.7 & 15.0 & 14.1 & 14.0 & 16.0 & 13.1 \\
Median & 90.0 & 80.0 & 81.0 & 83.5 & 81.0 & 80.0 \\
Minimum & 50.0 & 47.0 & 50.0 & 65.0 & 60.0 & 66.0 \\
Maximum & 140.0 & 130.0 & 110.0 & 120.0 & 130.0 & 120.0 \\
No. observed & 51 & 49 & 37 & 26 & 23 & 19 \\
\hline
\end{tabular}

creatinine taken every 12 months up to 60 months post-nephrectomy and the estimated creatinine clearance (MDRD) was calculated. There was a follow-up loss of $7.8 \%(4 / 51)$ of the patients.

Figure-4 shows the progress in renal function over the 60 months of observation. Twelve months post-nephrectomy $64 \%(30 / 47)$ of the patients presented an improvement in creatinine clearance (MDRD), represented by the ascending curves.

Figure- 5 shows the progress in renal function over 60 months. One can observe the 36\% (17/47) of patients whose renal function worsened (falling curves). Worthy of note among this group are the $23 \%$ of patients who initially lost renal function, but who demonstrated recovery of this function during the follow-up period. Dialysis was necessary in $8 \%$ $(4 / 51)$ of the patients over the 60 -month period.

When the characteristics of the patients divided into groups according to the improvement or worsening of their renal function were studied, they were found to be homogeneous, that is to say, without any statistically significant difference among the variables studied $(\mathrm{p}>0.05)$, i.e., these same factors as were analyzed for the blood pressure (Table-6).

Sixty percent of the cases (31/49) of this study had levels of serum rennin activity. Lateralization of the rennin was found in $85 \%$ of the patients divided among the different groups, with no statistical significance.

In eleven patients $(21.5 \%)$ there was no segmental stenosis of the vessel. In the histopathological analysis of the renal artery of the remaining patients we found atherosclerosis of the renal artery in $63 \%$ of the cases, fibromuscular dysplasia in $26 \%$ and Takayasu arteritis in 6\%. The histological data obtained from the renal parenchyma are: ischemic atrophy of the renal parenchyma in $72 \%$ of the cases, benign nephrosclerosis in 38\%; malignant nephro-

Table 4 -Differences in systolic and diastolic pressures as between the pre-operative period and each of the instants assessed in patients with $\mathrm{RVH}$ (in $\mathrm{mm} \mathrm{Hg}$ ).

\begin{tabular}{lcc}
\hline Months & $\Delta$ SAP $($ SAP post less SAP prev) & $\Delta$ DAP (DAP post less DAP prev) \\
\hline $12 \mathrm{~m}$ & $14.5 \pm 2.8(\mathrm{p}<0.001)$ & $9.7 \pm 2.4(\mathrm{p}<0.001) \mathrm{g}$ \\
$24 \mathrm{~m}$ & $8.5 \pm 3.9(\mathrm{p}=0.028)$ & $6.9 \pm 3.0(\mathrm{p}=0.023)$ \\
$36 \mathrm{~m}$ & $10.9 \pm 4.7(\mathrm{p}=0.022)$ & $7.1 \pm 3.5(\mathrm{p}=0.045)$ \\
$48 \mathrm{~m}$ & $10.1 \pm 5.2(\mathrm{p}=0.055)$ & $7.9 \pm 3.7(\mathrm{p}=0.035)$ \\
$60 \mathrm{~m}$ & $9.1 \pm 5.8(\mathrm{p}=0.117)$ & $7.6 \pm(4.0)(\mathrm{p}=0.062)$ \\
\hline
\end{tabular}




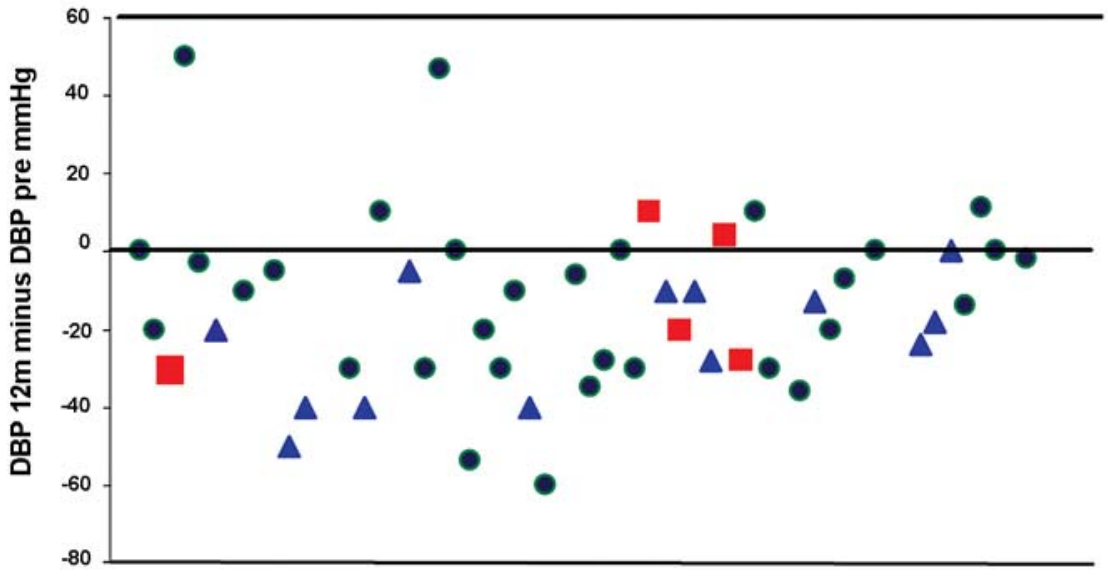

Figure 2 - Variation of SBP of each patient $12 \mathrm{~m}$ after nephrectomy $(n=49)$. • reduction, $\mathbf{\Delta}$ same and $\boldsymbol{\square}$ increased number of hypotensors.

sclerosis in $10 \%$, ischemic infarction of the renal parenchyma in $4 \%$ of the patients, interstitial nephritis in $6 \%$, chronic pyelonephritis in $12 \%$ and malignant renal neoplasms (of less than $1 \mathrm{~cm}$ ) in $4 \%$ of the cases studied.

In the group with improvement of the renal function, $54.2 \%$ of the patients had a diagnosis of fibromuscular dysplasia and $29.2 \%$ of the cases had atherosclerosis and by means of the application of the generalization of Fisher's Exact Test, a statisti- cally significant association was observed $(\mathrm{p}=0.003)$ between this histological type and the improvement in renal function. No embolic complications were identified in this series.

\section{COMMENTS}

RVH treatment has changed over recent years. The use of nephrectomy has declined as a result of the

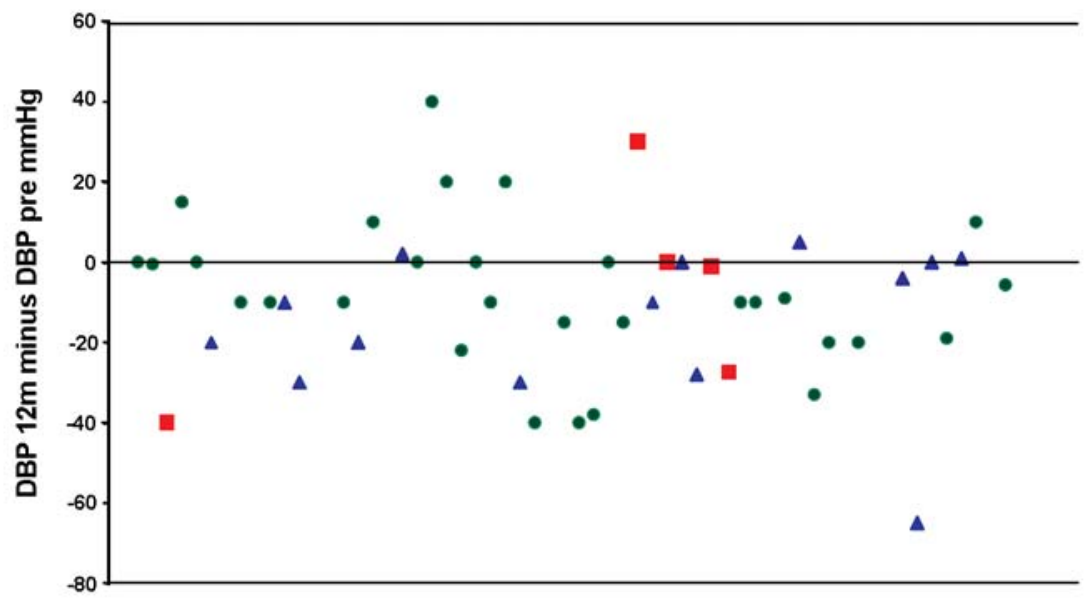

Figure 3 - Variation of the DBP of each patient 12 months after nephrectomy $(n=49)$. reduction, $\mathbf{\Delta}$ same and $\boldsymbol{\square}$ increase in the number of hypotensors. 
Table 5 - Characteristics of the patients with renovascular hypertension, in accordance with the pressure response to nephrectomy.

\begin{tabular}{lccc}
\hline \multicolumn{1}{c}{$\begin{array}{c}\text { Responders } \\
(\mathrm{N}=34)\end{array}$} & \multicolumn{2}{c}{$\begin{array}{c}\text { Non Responders } \\
(\mathrm{N}=15)\end{array}$} & p Value \\
\hline Age (years) & $46.2 \pm 13.7$ & $45.5 \pm 15.8$ & 0.891 \\
Sex: Male / Female & $35.3 \% / 64.7$ & $33.3 \% / 66.7 \%$ & 0.894 \\
Color: White / Non-white & $73.5 \% / 26.5 \%$ & $60.0 \% / 40.0 \%$ & 0.502 \\
Time of hypertension in years & $12(0.10-25)$ & $9(0.75-30)$ & 0.585 \\
Systolic Bood Pressure prev mm Hg & $149.1 \pm 23.0$ & $150.9 \pm 22.6$ & 0.800 \\
Diastolic Blood Pressure prev mm Hg & $92.7 \pm 14.7$ & $86.5 \pm 21.4$ & 0.247 \\
No. hypotensors & $2.9 \pm 0.9$ & $2.5 \pm 1.2$ & 0.267 \\
Serum Creatinine (mg/dL) & $1.1(0.8-4.5)$ & $1.5(0.8-4.4)$ & 0.116 \\
Clearance of Creatinine (mL/min)* & $60.5(15-100)$ & $44.0(17-83)$ & 0.095 \\
US atrophic kidney (cm) $\dagger$ & $7.3 \pm 1.2$ & $6.6 \pm 1.0$ & 0.061 \\
Lesion > 70\% of contralateral renal artery on Doppler & $35.3 \%$ & $40.0 \%$ & 0.753 \\
Scintigraphy com DTPA atrophic kidney \% & $9.6(0-49)$ & $5.0(0-17)$ & 0.115 \\
Contralateral kidney \% & $90.4(59-100)$ & $95.0(83-100)$ & 0.099 \\
Lateralization of serum rennin: Yes / No & $75.0 \% / 25 \%$ & $88.9 \% / 11.9 \%$ & 0.633 \\
Hypercholesterolemia: Yes / No & $61.8 \% / 38.2 \%$ & $73.3 \% / 26.7 \%$ & 0.433 \\
Smoking: Yes / No & $50.0 \% / 50.0 \%$ & $60.0 \% / 40.0 \%$ & 0.518 \\
Heart Disease: Yes / No & $29.4 \% / 70.6 \%$ & $33.3 \% / 66.7 \%$ & $>0.999$ \\
Stroke: Yes / No & $20.6 \% / 79.4 \%$ & $20.0 \% / 80.0 \%$ & $>0.999$ \\
Diabetes Mellitus :Yes / No & $17.6 \% / 82.4 \%$ & $13.3 \% / 86.7 \%$ & $>0.999$ \\
Peripheral Arterial Disease & & & $>0.999$ \\
Yes / No & $17.6 \% / 82.4 \%$ & $20.0 \% / 80.0 \%$ & $>0.999$ \\
Aortic Aneurism: Yes / No & $11.8 \% / 88.2 \%$ & $13.3 \% / 86.7 \%$ & \\
\hline
\end{tabular}

$\dagger U S=$ renal ultrasound

rise of new hypotensor drugs, developed on the basis of the knowledge the aldosterone angiotensin rennin system and the development of angioplasty techniques (PTA) with the implanting of "stents" $(3,11)$. More recently, the improved understanding of the mechanisms which harm dependent endothelial dilation and oxidative stress, especially as related to high levels of angiotensin II, particularly in the atherosclerotic lesion, herald new forms of treatment for the future (12).

The removal of the atrophic kidney caused by the severe stenosis of the renal artery was carried-out in patients with refractory arterial hypertension with no possibility of revascularization.

Various studies have been reported in the literature comparing percutaneous revascularization and conservative clinical treatment in the treatment of RVH (13), but there are few series regarding the nephrectomy of the atrophic kidney as an alternative form of treatment for the bearer of renovascular hypertension with blood pressure of difficult control (14-16).

This study analyzed 51 nephrectomies of the atrophic kidney for complete obstruction of the 


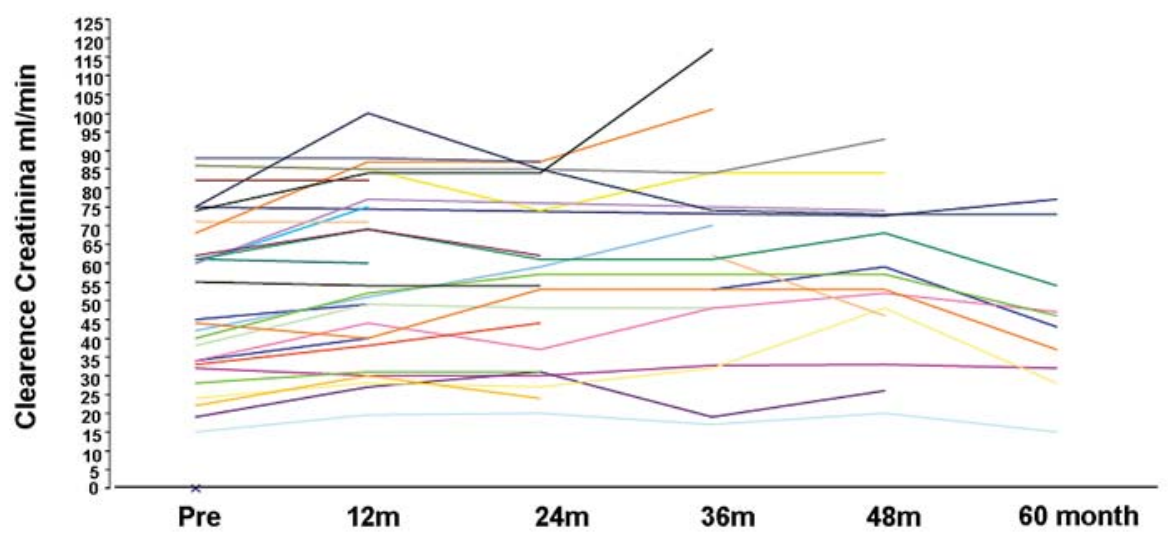

Figure 4 - Patients with improved creatinine clearance $(n=30)$.

renal artery. There was a predominance of the video laparoscopic technique, which was used in $70.5 \%$ of the cases. A year after nephrectomy, SBP was $135 \pm$ $21 \mathrm{~mm} \mathrm{Hg}$ and DBP of $81 \pm 15 \mathrm{~mm} \mathrm{Hg}$, significantly lower than the initial levels, and they were thus maintained for 36 months and 48 months, respectively, together with a significant reduction in the number of hypotensors.

The division into groups on the basis of response in terms of blood pressure and renal function one year after nephrectomy, was performed to identify those characteristics which determined the response. Sixty-eight percent of the cases which presented an improvement in arterial hypertension were included in the group (R), $8 \%$ of them being cured after one year, 75\% (3/4) of them being patients with RHV due to fibromuscular dysplasia of the renal artery and $32 \%$ of the remaining cases belonging to the NR group (Table-5). The creatinine clearance (MDRD) curve $(9,10,13)$ was used to enable us to classify the patients by group into those which presented either improvement or worsening of the renal function at 12 months. There was an improvement in creatinine clearance one year after nephrectomy in $64 \%$ of the cases (Figure- 4 ) and a worsening in $36 \%$ (Figure-5). Twelve percent of the

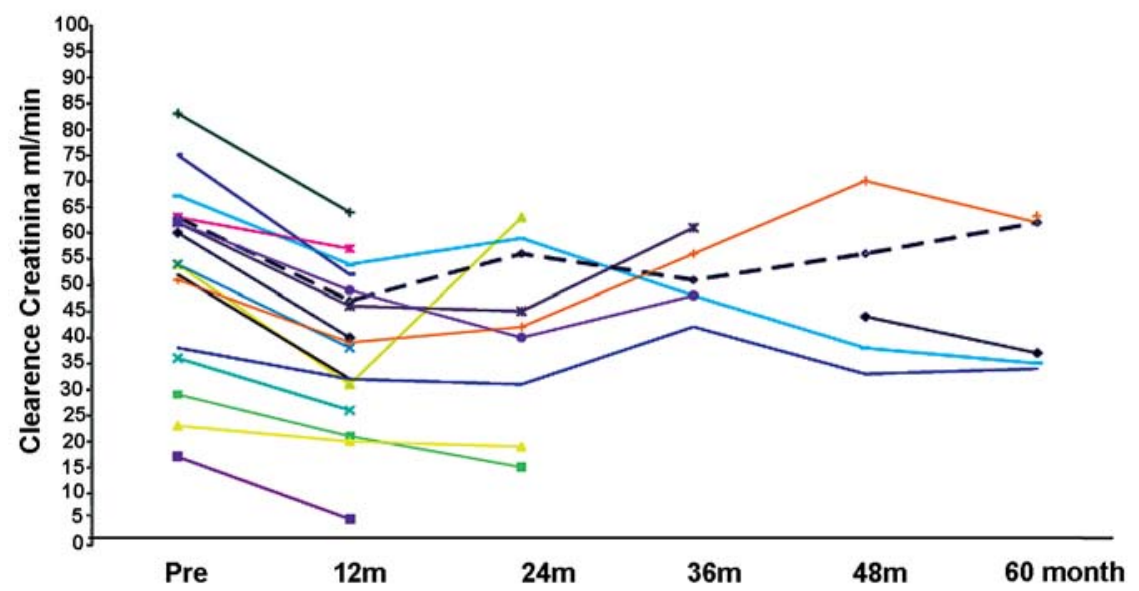

Figure 5 - Patients with a worsening of creatinine clearance $(n=17)$. 
Table 6 - Characteristics of the patients in accordance with the response of their renal function to nephrectomy.

\begin{tabular}{lccc}
\hline \multicolumn{1}{c}{\begin{tabular}{c} 
Improvement \\
\multicolumn{1}{c}{$(\mathrm{N}=30)$}
\end{tabular}} & \multicolumn{2}{c}{ Worsening } \\
$(\mathrm{N}=17)$ & p Value \\
\hline Age (years) & $47.1 \pm 15.3$ & $46.1 \pm 14.3$ & 0.825 \\
Sex: Male / Female & $33.3 \% / 66.7 \%$ & $35.3 \% / 64.7 \%$ & 0.892 \\
Color: White / Non-white & $80.0 \% / 20.0 \%$ & $52.9 \% / 47.1 \%$ & 0.051 \\
Time of hypertension years & $8.5(0.10-30)$ & $13.0(0.75-30)$ & 0.337 \\
SBP previous mmHg & $148.2 \pm 23.3$ & $148.5 \pm 23.2$ & 0.963 \\
DBP previous mm Hg & $90.7 \pm 16.4$ & $91.8 \pm 15.9$ & 0.824 \\
$\mathrm{~N}^{\circ}$. hypotensors & $2.8 \pm 1$ & $2.8 \pm 1$ & 0.939 \\
Serum Creatinine mg/dL & $1.3(0.8-4.5)$ & $1.3(0.8-4.4)$ & 0.991 \\
Clearance Creatinine mL/min* & $57(15-100)$ & $54(17-83)$ & 0.903 \\
US atrophic kidney cm† & $7.1 \pm 1.0$ & $7.1 \pm 1.3$ & 0.929 \\
Lesion > $70 \%$ of contra-lat. renal artery on Doppler & $43.3 \%$ & $17.6 \%$ & 0.074 \\
Scintigraphy with DTPA atrophic kidney $\%$ & $9(0-49)$ & $7(0-28)$ & 0.649 \\
Contralateral kidney \% & $91(59-100)$ & $93(72-100)$ & 0.600 \\
Lateralization of serum rennin: Yes / No & $78.9 \% / 21.1 \%$ & $70.0 \% / 30.0 \%$ & 0.665 \\
Hypercholesterolemia: Yes / No & $60.0 \% / 40.0 \%$ & $82.4 \% / 17.6 \%$ & 0.114 \\
Smoking: Yes / No & $43.3 \% / 56.7 \%$ & $64.7 \% / 35.3 \%$ & 0.159 \\
Heart disease: Yes / No & $33.3 \% / 66.7 \%$ & $29.4 \% / 70.6 \%$ & 0.782 \\
Diabetes Mellitus: Yes / No & $13.3 \% / 86.7 \%$ & $17.6 \% / 82.4 \%$ & 0.692 \\
Stroke: Yes / No & $13.3 \% / 86.7 \%$ & $29.4 \% / 70.6 \%$ & 0.252 \\
Aortic Aneurism: Yes / No & $6.7 \% / 93.3 \%$ & $17.6 \% / 82.4 \%$ & 0.336 \\
\hline
\end{tabular}

$\digamma U S=$ renal ultrasound

patients with creatinine clearance $<30 \mathrm{~mL} / \mathrm{min}$ at surgery, continued not to need dialysis during the period of 60 months analyzed (Figure-4) and 8.5\% of the patients whose renal function worsened at 12 months, showed recovery of the function over the 60 months (Figure-5). There was no statistically significant lesion present (Table-6). Moderate renal insufficiency, which might be an important factor leading to a final need for dialysis, was not significant in this patient population, possibly because of the small number of cases involved.

In the four groups, the characteristics studied, such as sex, color, age, smoking, hypercholesterolemia and co-morbidities presented similar distribution with no significant statistical difference $(\mathrm{p}>0.095)$.
No lateralization of the rennin was found as a predicative factor of results, similar data are described by Hasbak et al. (17). The factors which would explain the absence of the lateralization of rennin are bilateral stenosis of the renal artery, states of volemic depletion, chronic use of inhibitors of the conversion enzyme of angiotensin I to II (IECA) and renal insufficiency. Rossi et al. (18) found a correlation of the lateralization of serum rennin activity only in bearers of RVH with unilateral atrophic kidney by complete occlusion of the renal artery that underwent nephrectomy, but there was no lateralization of the rennin plasma in patients treated with angioplasty.

The presence of significant lesion (> 70\%) of the contra-lateral renal artery made no statistically 
significant difference $(\mathrm{p}=0.753)$ to the results of this study. Bilateral lesion was treated by nephrectomy after revascularization of the contra-lateral renal artery in $27 \%$ (14 patients).

Kane et al. (14) also found an improvement in blood pressure in patients who had undergone nephrectomy of the atrophic kidney (59\%) for the treatment of RVH. In the comparison, in nephrectomy with the revascularization of the ischemic kidney undertaken by Oskin et al. (13) to evaluate blood pressure and renal function, nephrectomy was as effective as revascularization for the control of arterial hypertension, but was inferior to revascularization for the preservation of the renal function.

The average age of 47 years in this series is inferior to the 65 years of other reported series $(3,13,14)$. Of the $34(67 \%)$ women in this study, 38\% were of childbearing age, at risk of becoming pregnant. The prevalence of renovascular hypertension during pregnancy is unknown, whereas the significantly greater risk of pre-eclampsia in the woman with chronic arterial hypertension is known. Nephrectomy of the atrophic kidney before a new pregnancy after a failure, can improve the maternal and fetal prognosis (19).

The relationship between stenosis of the renal artery and other systemic lesions is well known (2022). The significant prevalence of stenosis of the renal artery (greater than 75\%) in patients who died from stroke was $10.4 \%$ of the cases, $4.6 \%$ of whom with complete occlusion of the vessel (20). In the literature, patients with peripheral vascular disease associated with stenosis of the atherosclerosclerotic renal artery suffered higher mortality from cardiac causes during the two-year follow-up (21) and the severity of the lesion of the renal artery was directly proportional to the worst cardiac prognosis (22).

The exact relationship between the degree of stenosis and the loss of mass is unknown. As the Doppler is not an invasive examination it has been used for the follow-up of stenotic lesions. Significant stenosis $(>60 \%)$ and severe arterial hypertension, progressed with $36 \%$ of renal atrophy over two years, followed-up using Doppler examination(23).

Arteriography was the examination primarily used to determine the presence of the obstruction and the viability of the atrophic kidney. Presently, magnetic resonance angiography and angiotomography of the renal arteries may be used as alternatives to arteriography with the advantage of avoiding arterial catheterization $(24,25)$. However, when there is a suspicion of fibrodysplasia of the renal artery, where the lesions are more distal, magnetic resonance angiography is not appropriate, as it has greater sensitivity to the lesions of the artery located up to $3 \mathrm{~cm}$ from the ostium $(24,25)$.

In this series, among the causes of the renal atrophy which led to nephrectomy, we found atherosclerosis of the renal artery, fibromuscular dysplasia, trauma of the renal artery, thrombosis of the renal artery in patients submitted to renal autotransplant and failure of endovascular revascularization. In another series, the nephrectomy of the atrophic kidney cured the RVH caused by a fall which occurred when removing the traumatic lesion from the renal artery (26). The high incidence of thrombosis is due to the fact that the renal arteries in these cases are small, difficult to manipulate and possess lesions aggravated by previous procedures (16).

The diagnosis of fibromuscular dysplasia of the renal artery was associated with better results as regards both pressure and renal function, renal dialytic insufficiency being a rare cause (27).

From the physiological point of view, the reduction in the levels of angiotensin II after the nephrectomy of the atrophic kidney would cause lesser oxidative stress and could lead to the restoration of the dependent endothelial dilation, responsible in the final analysis for the vascular lesions (renal, coronary and cerebral), mainly those of atherosclerotic etiology. It is known that high levels of angiotensin II are related to an increase in the pro-fibrotic factors, with the consequent replacement of the functioning glomerulus by fibrosis, leading to a loss of renal mass and the advance of renal insufficiency. Hypercholesterolemia, especially the increase in the oxidized LDL, associated with stenosis of the renal artery, speeds up the development of fibrosis in the ischemic kidney by the spread of pro-fibrotic mechanisms (NF- $\beta$, TGF- $\beta$, oxidative stress among others) and suppression of the remodeling of the tissues (28).

In the future, we should have more options for the treatment of arterial hypertension and vascular obstruction. New therapies such as gene therapy and cell therapy, which do not call for the use of drugs, are 
appearing. The implantation of embryonic cells (stem cells) or of genes which codify angiogenic factors may be used as alternative therapies for the inoperable vascular obstructions. Gene therapy takes endothelial growth factors or blocks the harmful genes involved in the pathogenesis of the disease. The endothelial dysfunction caused by oxidative stress, an important mechanism of the atherosclerotic vascular lesion, may be treated in the future either by cell therapy or by gene therapy in association with pharmacological therapy $(29,30)$.

\section{CONCLUSION}

In patients with renovascular hypertension, nephrectomy of the atrophic kidney is a procedure which results in improvement of the arterial hypertension and of the renal function in two-thirds of patients.

\section{CONFLICT OF INTEREST}

None declared.

\section{REFERENCES}

1. Safian RD, Textor SC: Renal-artery stenosis. N Engl J Med. 2001; 344: 431-42.

2. Slovut DP, Olin JW: Fibromuscular dysplasia. N Engl J Med. 2004; 350: 1862-71.

3. Garovic VD, Textor SC: Renovascular hypertension and ischemic nephropathy. Circulation. 2005; 112: 1362-74.

4. Rimmer JM, Gennari FJ: Atherosclerotic renovascular disease and progressive renal failure. Ann Intern Med. 1993; 118: 712-9.

5. Hansen KJ, Edwards MS, Craven TE, Cherr GS, Jackson SA, Appel RG, et al.: Prevalence of renovascular disease in the elderly: a population-based study. J Vasc Surg. 2002; 36: 443-51.

6. García-Donaire JA, Alcázar JM: Ischemic nephropathy: detection and therapeutic intervention. Kidney Int Suppl. 2005; 68: S131-6.

7. Loscalzo J: Nitric oxide and vascular disease. N Engl J Med. 1995; 333: 251-3.
8. Seelig HP: The Jaffe reaction with creatinine. Z Klin Chem Klin Biochem. 1969;:18: 30-9.

9. Rundback JH, Sacks D, Kent KC, Cooper C, Jones D, Murphy T, et al.: Guidelines for the reporting of renal artery revascularization in clinical trials. American Heart Association. Circulation. 2002; 106: 1572-85.

10. Poggio ED, Wang X, Weinstein DM, Issa N, Dennis VW, Braun WE, et al.: Assessing glomerular filtration rate by estimation equations in kidney transplant recipients. Am J Transplant. 2006; 6: 100-8.

11. Gray BH: Intervention for renal artery stenosis: endovascular and surgical roles. J Hypertens Suppl. 2005; 23: S23-9.

12. Higashi Y, Sasaki S, Nakagawa K, Matsuura H, Oshima T, Chayama K: Endothelial function and oxidative stress in renovascular hypertension. N Engl J Med. 2002; 346: 1954-62.

13. Krumme B, Donauer J: Atherosclerotic renal artery stenosis and reconstruction. Kidney Int. 2006; 70: 1543-7.

14. Oskin TC, Hansen KJ, Deitch JS, Craven TE, Dean RH: Chronic renal artery occlusion: nephrectomy versus revascularization. J Vasc Surg. 1999; 29: 140-9.

15. Kane GC, Textor SC, Schirger A, Garovic VD: Revisiting the role of nephrectomy for advanced renovascular disease. Am J Med. 2003; 114: 729-35.

16. Geyskes GG, Oei HY, Klinge J, Kooiker CJ, Puylaert CB, Dorhout Mees EJ: Renovascular hypertension: the small kidney updated. Q J Med. 1988; 66: 203-17.

17. Hasbak P, Jensen LT, Ibsen H; East Danish Study Group on Renovascular Hypertension: Hypertension and renovascular disease: follow-up on 100 renal vein renin samplings. J Hum Hypertens. 2002; 16: 27580.

18. Rossi GP, Cesari M, Chiesura-Corona M, Miotto D, Semplicini A, Pessina AC: Renal vein rennin measurements accurately identify renovascular hypertension caused by total occlusion of renal artery. J Hypertension. 2002; 5: 975-84.

19. Thorsteinsdottir B, Kane GC, Hogan MJ, Watson WJ, Grande JP, Garovic VD: Adverse outcomes of renovascular hypertension during pregnancy. Nat Clin Pract Nephrol. 2006; 2: 651-6.

20. Kuroda S, Nishida N, Uzu T, Takeji M, Nishimura M, Fujii T, et al.: Prevalence of renal artery stenosis in autopsy patients with stroke. Stroke. 2000; 31: 61-5.

21. Pillay WR, Kan YM, Crinnion JN, Wolfe JH; Joint Vascular Research Group, UK: Prospective multicentre study of the natural history of atherosclerotic renal artery stenosis in patients with peripheral vascular disease. Br J Surg. 2002; 89: 737-40. 
22. Conlon PJ, Little MA, Pieper K, Mark DB: Severity of renal vascular disease predicts mortality in patients undergoing coronary angiography. Kidney Int. 2001; 60: $1490-7$.

23. Caps MT, Zierler RE, Polissar NL, Bergelin RO, Beach $\mathrm{KW}$, Cantwell-Gab K, et al.: Risk of atrophy in kidneys with atherosclerotic renal artery stenosis. Kidney Int. 1998; 53: 735-42.

24. Pedersen EB: New tools in diagnosing renal artery stenosis. Kidney Int. 2000; 57: 2657-77.

25. Lee V: The 89 th. Annual Scientific Assembly and Annual Meeting of Radiological Society of North America. Renal MRI: Novel techniques and contrast agents.2006 [Access in 2006 Jan 06]. Available at: www.medscape.com

26. Negoro H, Iwamura H, Oka H, Kawakita M, Ariyoshi $\mathrm{K}$, Koda Y, et al.: Traumatic renal artery thrombosis with renovascular hypertension. Int J Urol. 2004; 11: 903-5.
27. Mounier-Vehier C, Lions C, Jaboureck O, Devos P, Haulon S, Wibaux M, et al.: Parenchymal consequences of fibromuscular dysplasia renal artery stenosis. Am J Kidney Dis. 2002; 40: 1138-45.

28. Chade AR, Rodriguez-Porcel M, Grande JP, Zhu X, Sica V, Napoli C, et al.: Mechanisms of renal structural alterations in combined hypercholesterolemia and renal artery stenosis. Arterioscler Thromb Vasc Biol. 2003; 23: 1295-301.

29. Higashi Y, Nishioka K, Umemura T, Chayama K, Yoshizumi M: Oxidative stress, endothelial function and angiogenesis induced by cell therapy and gene therapy. Curr Pharm Biotechnol. 2006; 7: 109-16.

30. Melo LG, Gnecchi M, Pachori AS, Kong D, Wang K, Liu X, Pratt RE, Dzau VJ: Endothelium-targeted gene and cell-based therapies for cardiovascular disease. Arterioscler Thromb Vasc Biol. 2004; 24: 1761-74.

Accepted after revision:

October 18, 2009

\section{Correspondence address:}

Dr. Myrian J. Thomaz

Department of Urology

Faculty of Medicine, University of Sao Paulo

Sao Paulo, Brazil

E-mail:m.j.thomaz@uol.com.br 